\title{
Myoferlin Expression and Its Correlation with FIGO Histologic Grading in Early-Stage Endometrioid Carcinoma
}

Min Hye Kim ${ }^{1} \cdot$ Dae Hyun Song ${ }^{2,3,4}$ Gyung Hyuck Ko ${ }^{1,2,3}$ · Jeong Hee Lee ${ }^{1,2,3}$ Dong Chul Kim ${ }^{1,2,3} \cdot$ Jung Wook Yang ${ }^{1}$ Hyang Im Lee ${ }^{4}$ Hyo Jung $\mathrm{An}^{4}$ Jong Sil Lee ${ }^{1,2,3}$

'Department of Pathology, Gyeongsang National University Hospital, Jinju; ${ }^{2}$ Gyeongsang National University School of Medicine, Jinju; ${ }^{3}$ Gyeongsang Institute of Health Science, Jinju; ${ }^{4}$ Department of Pathology, Gyeongsang National University Changwon Hospital, Changwon, Korea

Received: August 29, 2017

Revised: November 7, 2017

Accepted: November 29, 2017

\section{Corresponding Author}

Jong Sil Lee, MD

Department of Pathology, Gyeongsang National

University School of Medicine, 15 Jinju-daero

816beon-gil, Jinju 52727, Korea

Tel: +82-55-750-8231

Fax: $+82-55-759-7952$

E-mail: jongsil25@gnu.ac.kr

\begin{abstract}
Background: For endometrioid carcinoma patients, International Federation of Gynecologists and Obstetricians (FIGO) histologic grading is very important for identifying the appropriate treatment method. However, the interobserver discrepancy with this three-tiered grading system is a serious potential problem. In this study, we used immunohistochemistry to analyze the relationship between FIGO histologic grading score and myoferlin expression. Methods: We studied the endometrioid carcinoma tissues of 60 patients from Gyeongsang National University Hospital between January 2002 and December 2009. Immunohistochemical analysis of myoferlin was performed on tissue microarray blocks from surgical specimens. Results: Myoferlin expression was observed in 58 of 60 patients. Moderate and strong myoferlin expression was observed in lowgrade endometrioid carcinoma, while there was a tendency toward loss of myoferlin expression in high-grade endometrioid carcinoma $(p<.001)$. Conclusions: Our study revealed that myoferlin loss is significantly correlated with high FIGO grade of endometrioid carcinoma.
\end{abstract}

Key Words: Myoferlin protein; Carcinoma, Endometrioid; FIGO grade; Immunohistochemistry; Chemotherapy
In endometrioid carcinoma patients, International Federation of Gynecologists and Obstetricians (FIGO) histologic grading is very important in determining the treatment method and predicting the patient's prognosis. ${ }^{1-3}$ Particularly, it is recommended that patients with FIGO stage I and histologic grade 3 endometrioid carcinoma undergo adjuvant radiotherapy after surgery, ${ }^{3}$ while patients with FIGO stage I and histologic grade 1 or 2 endometrioid carcinoma should be treated with surgery only. However, the interobserver discrepancy of this three-tiered FIGO grading system can pose a serious potential problem. Thus, many authors have used immunohistochemical (IHC) staining, ${ }^{4-6}$ genetic molecular testing, ${ }^{7-9}$ and other two-tiered systems ${ }^{10,11}$ to reduce the discrepancy.

Myoferlin, a protein in the cellular membrane, is involved in cellular regeneration after injury. ${ }^{12}$ Recent studies have reported a correlation between the prognosis of cancer patients and myoferlin expression in breast, lung, oropharyngeal, and pancreatic cancer. ${ }^{13-16}$
In this study, we used IHC staining to analyze the relationship between FIGO histologic grading and myoferlin expression.

\section{MATERIALS AND METHODS}

\section{Case selection}

We collected clinical data from the charts of endometrioid carcinoma patients treated at Gyeongsang National University Hospital, Jinju, Korea, between January 2002 and December 2009. A total of 60 patients who underwent hysterectomy were enrolled. The tumor stage and histologic grade of each case were assessed using the FIGO system. All gross photographs and hematoxylin and eosin-stained glass slides of surgical specimens were reviewed by two pathologists.

This study was approved by the Institutional Review Board of Gyeongsang National University Hospital with a waiver of informed consent (IRB No. GNUH-2015-12-003). 


\section{Tissue microarray}

Tumor samples were fixed overnight in 20\% neutral-buffered formalin and were examined grossly, dissected, and embedded in paraffin blocks. One or two representative blocks were selected by microscopic examination. One representative core $(3 \mathrm{~mm}$ in diameter) tissue was obtained from each paraffin block and arranged in new recipient tissue microarray blocks. Representative areas of the donor blocks were selected from near the invasive front.

\section{$\mathrm{IHC}$ analysis}

A primary antibody for myoferlin (1:100, 7D6, Abcam, Cambridge, UK) was used to investigate protein expression. The IHC method used was described in detail in our previous report. ${ }^{14}$

The positive control for myoferlin was normal endometrial tissue. Tissues showing an intensity equal to or stronger than that of normal endometrial tissue were classified as grade 3. The IHC slides were scored using the three-tiered FIGO system by two pathologists.

\section{Statistical analysis}

Correlation analyses were performed using the chi-square test. A p-value of $<.05$ was considered statistically significant. All statistics were analyzed using SPSS ver. 24.0 (IBM Corp., Armonk, NY, USA).

\section{RESULTS}

\section{Clinicopathological features of the patients}

The clinicopathological features of 60 endometrioid carcinoma patients are summarized in Table 1. The mean age was 51 years. All patients underwent hysterectomy. Among 60 cases, 43 (71.7\%) were classified as FIGO stage Ia after surgery, whereas 40 $(66.7 \%)$ were considered FIGO histologic grade 1.

\section{Myoferlin expression in nontumorous endometrial tissue}

Nontumorous endometrial tissues were obtained from leiomyoma or adenomyosis patients who underwent hysterectomy. In normal epithelial tissues, moderate and strong myoferlin expression was observed. Specifically, endometrial tissue in secretory phase showed strong expression (Fig. 1).

\section{Myoferlin expression in endometrioid carcinoma}

The clinicopathological features of the cases included in this study are summarized in Table 1. Myoferlin expression in the cytoplasm and cellular membrane of cancer cells was observed in 58 of the 60 patients (Fig. 1). Similarly, cytoplasmic and membra-
Table 1. Clinical and pathological features of 60 endometrioid carcinoma patients

\begin{tabular}{lc}
\hline Variable & No. of patients (\%) \\
\hline Mean age (yr) & $51(35-78)$ \\
T category & \\
la & $43(71.7)$ \\
Ib & $12(20)$ \\
II & $3(5)$ \\
IIla & $1(1.7)$ \\
IIIb & $1(1.7)$ \\
Histologic grade & \\
1 & $40(66.7)$ \\
2 & $15(25)$ \\
3 & $5(8.3)$ \\
Myoferlin expression & \\
Negative & $2(3.3)$ \\
Weak (+1) & $4(6.6)$ \\
Moderate (+2) & $9(15)$ \\
Strong (+3) & $45(75)$ \\
Total & 60 \\
\hline
\end{tabular}

nous expression of myoferlin was evident in normal endometrial glands in proliferative and secretory phases. Forty-five patients $(75 \%)$ exhibited strong myoferlin expression, similar to that in normal endometrial glands.

\section{Correlation between myoferlin expression and FIGO histologic grading}

The correlation is described in Table 2. In the chi-square test, moderate and strong myoferlin expression was observed in lowgrade endometrioid carcinoma, while there was a tendency toward loss of myoferlin expression in high-grade endometrioid carcinoma $(\mathrm{p}<.001)$.

\section{Correlation between myoferlin expression and FIGO staging}

The correlation is shown in Table 3. In the chi-square test, most patients with FIGO stage III endometrioid carcinoma exhibited negative or weak myoferlin expression $(+1)$. A tendency toward loss of myoferlin expression was also observed in late-stage endometrioid carcinoma $(\mathrm{p}<.001)$.

\section{DISCUSSION}

Myoferlin is a little known protein. To our knowledge, this study is the first to identify myoferlin expression in endometrial tissue. Recent studies have reported that myoferlin contributes to the proliferation, migration, and invasion of cancer cells and is overexpressed in several types of cancer. However, in this study, endometrial cancer showed the opposite result; myoferlin expression was decreased in high-grade endometrioid carcinoma, probably 

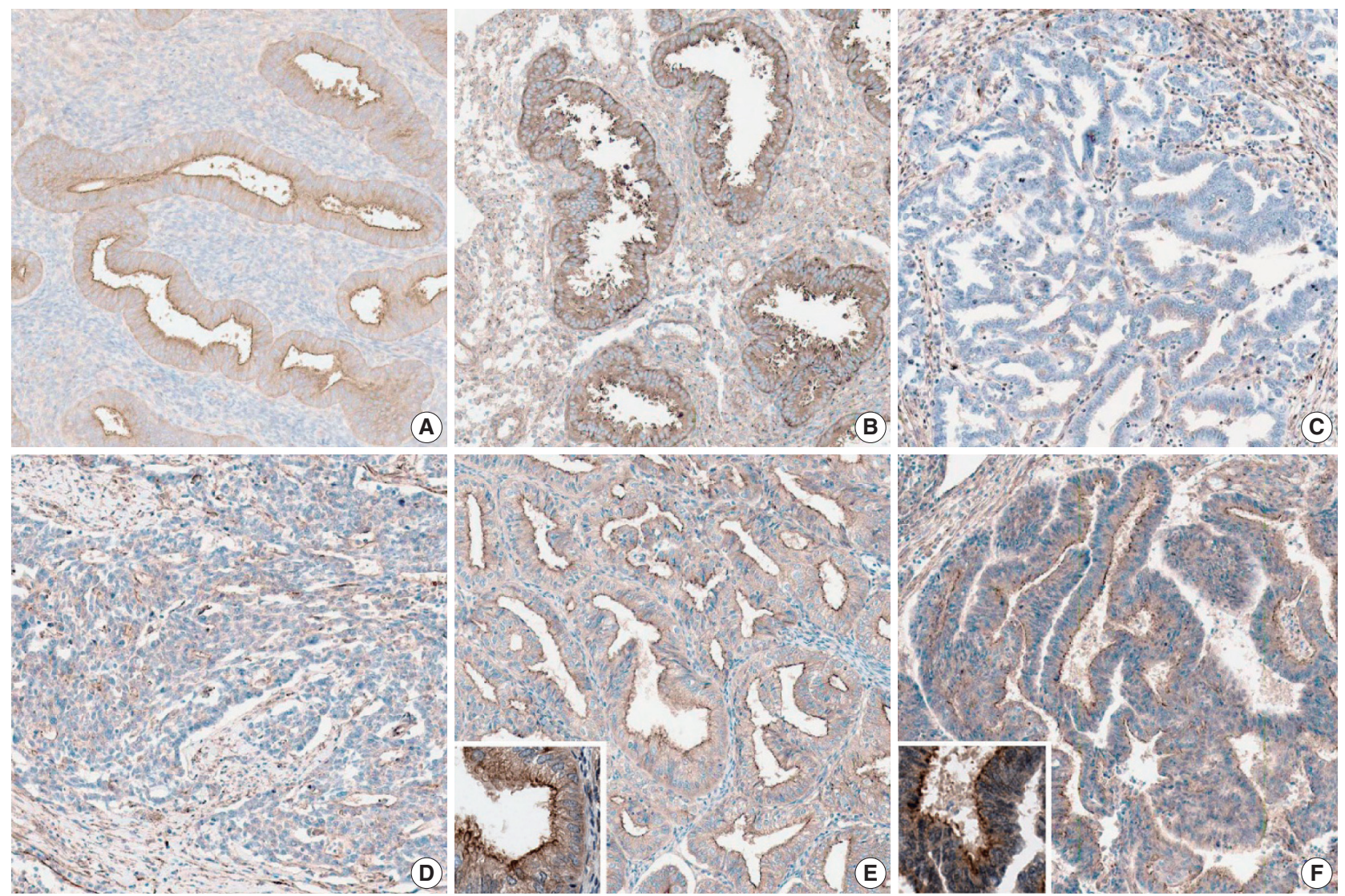

Fig. 1. Myoferlin expression in nontumorous endometrial tissue in proliferative phase (A) and secretory phase (B). (C) Loss of myoferlin expression in high-grade endometrioid carcinoma. Weak (+1, International Federation of Gynecologists and Obstetricians [FIGO] grade 3) (D), moderate $(+2$, FIGO grade 2) $(\mathrm{E})$, and strong $(+3$, FIGO grade 1) (F) expression of myoferlin in endometrioid carcinoma.

Table 2. Correlation between myoferlin expression and FIGO histologic grading

\begin{tabular}{lrcc}
\hline Histologic grade & $\leq+1$ & $\geq+2$ & Total \\
\hline 1 & 1 & 39 & 40 \\
2 & 2 & 13 & 15 \\
3 & 37 & 2 & 5 \\
Total & 6 & 54 & \\
\hline
\end{tabular}

\section{$p<.001$}

FIGO, International Federation of Gynecologists and Obstetricians.

because normal endometrial tissue undergoes a continuous cycle of regeneration. Moreover, myoferlin has been reported to be involved in cellular regeneration after injury. ${ }^{14}$ The tumorigenesis of endometrioid carcinoma is associated with noncyclic continuous exposure to sex hormones. Our results implicate a correlation among cellular regeneration, hormonal effect, and myoferlin expression.

Though FIGO grading is important in guiding patient treatment, the interobserver discrepancy of this three-tiered grading system is a potential problem. Hence, many authors have attempted grading using IHC staining, ${ }^{4-6}$ genetic molecular testing, ${ }^{7-9}$ other two-tiered systems, ${ }^{10,11}$ and curettage and cytology ${ }^{17}$ to decrease
Table 3. Correlation between myoferlin expression and FIGO staging

\begin{tabular}{|c|c|c|c|}
\hline \multirow{2}{*}{ T category } & \multicolumn{2}{|c|}{ Myoferlin } & \multirow{2}{*}{ Total } \\
\hline & $\leq+1$ & $\geq+2$ & \\
\hline la & 1 & 42 & 43 \\
\hline Ib & 3 & 9 & 12 \\
\hline$\|$ & 0 & 3 & 3 \\
\hline Illa & 1 & 0 & 1 \\
\hline Illb & 1 & 0 & 1 \\
\hline Total & 6 & 54 & \\
\hline
\end{tabular}

$\mathrm{p}<.001$.

FIGO, International Federation of Gynecologists and Obstetricians.

discrepancies.

In a previous study, Daniilidou et al. ${ }^{5}$ investigated PTEN and $p 53$ gene expression in endometrioid and serous papillary carcinoma and showed that these biomarkers contribute to accurate diagnosis and therapeutic decisions in relation to tumor stage and grade. In another notable study on the cytologic scoring of endometrioid carcinoma of the endometrium, Nishimura et al..$^{17}$ examined 64 cytologic samples and scored them using 10 cytology character- 
istics. The cytologic grade was closely related to histologic grade, and a high cytologic score was correlated with $p 53$ mutation and myometrial invasion. The study implied that the cytologic scoring system for endometrioid carcinoma is useful for predicting histologic grade and malignant potential of the tumor.

Guan et al..$^{10}$ tried a new binary grading system for endometrial carcinoma and compared it with an existing binary grading system and FIGO grading in hysterectomy specimens. They examined 254 hysterectomies and graded them according to the new grading system including architecture pattern and nuclear atypia. They concluded that the three-tiered FIGO grading system retained superior prognostic power. However, the new binary grading system is an attractive option due to its good reproducibility and the elimination of ambiguity of intermediate grades.

In the present study, we also tried to decrease discrepancies by determining the FIGO grade according to myoferlin expression. Our statistically significant $(\mathrm{p}<.001)$ finding was that moderate and strong myoferlin expression was observed in low-grade endometrioid carcinoma, and loss of myoferlin expression was noted in high-grade endometrioid carcinoma. Here, we found that myoferlin could be a valuable marker for the accurate grading of uterine endometrioid carcinoma.

In this study, the level of myoferlin expression in endometrioid carcinoma was opposite that observed in other studies, suggesting a hidden mechanism underlying the continuous regeneration of tissue in the endometrium. Thus, further investigation in the role of myoferlin in the endometrium and in tumorigenesis of endometrioid carcinoma is recommended.

This study had some limitations. The number of patients involved was only 60 , and the cases consisted of mostly early-stage endometrioid carcinoma. Therefore, further studies with larger sample sizes are necessary to validate the significance of myoferlin expression in early- and late-stage endometrioid carcinoma.

In conclusion, our study revealed that myoferlin loss is significantly correlated with high FIGO grade of endometrioid carcinoma. To our knowledge, this is the first report on myoferlin expression in endometrial tissue, and our results could help in the management of patients with endometrioid carcinoma.

\section{Conflicts of Interest}

No potential conflict of interest relevant to this article was reported.

\section{Acknowledgments}

This work was supported by the Development Fund Founda- tion, Gyeongsang National University (2015).

\section{REFERENCES}

1. Dvalishvili I, Charkviani L, Turashvili G, Burkadze G. Clinical characteristics of prognostic factors in uterine endometrioid adenocarcinoma of various grade. Georgian Med News 2006; (132): 24-7.

2. Zaki MA, Robbins JR, Fatteh S, Mahan MG, Hanna RK, Elshaikh MA. Histological grade predicts for recurrence in patients with uterine endometrioid carcinoma without myometrial involvement. Anticancer Res 2012; 32: 4061-5.

3. Creasman WT, Odicino F, Maisonneuve P, et al. Carcinoma of the corpus uteri. FIGO 26th Annual Report on the Results of Treatment in Gynecological Cancer. Int J Gynaecol Obstet 2006; 95 Suppl 1: S105-43.

4. Apostolou G, Apostolou N, Biteli M, Kavantzas N, Patsouris E, Athanassiadou P. Utility of Ki-67, p53, Bcl-2, and Cox-2 biomarkers for low-grade endometrial cancer and disordered proliferative/ benign hyperplastic endometrium by imprint cytology. Diagn Cytopathol 2014; 42: 134-42.

5. Daniilidou K, Frangou-Plemenou M, Grammatikakis J, Grigoriou O, Vitoratos N, Kondi-Pafiti A. Prognostic significance and diagnostic value of PTEN and p53 expression in endometrial carcinoma: a retrospective clinicopathological and immunohistochemical study. J BUON 2013; 18: 195-201.

6. Kounelis S, Kapranos N, Kouri E, Coppola D, Papadaki H, Jones MW. Immunohistochemical profile of endometrial adenocarcinoma: a study of 61 cases and review of the literature. Mod Pathol 2000; 13: $379-88$

7. Abdalla N, Piorkowski R, Stanirowski P, Slomka A, Cendrowski K, Sawicki W. Assessment of levels of the tumor markers HE4 and CA125 considering staging, grading and histological types of endometrial cancer. Prz Menopauzalny 2016; 15: 133-7.

8. Coenegrachts L, Schrauwen S, Van Bree R, et al. Increased expression of placental growth factor in high-grade endometrial carcinoma. Oncol Rep 2013; 29: 413-8.

9. Kashima H, Wu RC, Wang Y, et al. Laminin C1 expression by uterine carcinoma cells is associated with tumor progression. Gynecol Oncol 2015; 139: 338-44.

10. Guan H, Semaan A, Bandyopadhyay S, et al. Prognosis and reproducibility of new and existing binary grading systems for endometrial carcinoma compared to FIGO grading in hysterectomy specimens. Int J Gynecol Cancer 2011; 21: 654-60.

11. Kapucuoglu N, Bulbul D, Tulunay G, Temel MA. Reproducibility of grading systems for endometrial endometrioid carcinoma and their relation with pathologic prognostic parameters. Int J Gynecol 
Cancer 2008; 18: 790-6

12. Leung C, Yu C, Lin MI, Tognon C, Bernatchez P. Expression of myoferlin in human and murine carcinoma tumors: role in membrane repair, cell proliferation, and tumorigenesis. Am J Pathol 2013; 182: 1900-9.

13. Blomme A, Costanza B, de Tullio $\mathrm{P}$, et al. Myoferlin regulates cellular lipid metabolism and promotes metastases in triple-negative breast cancer. Oncogene 2017; 36: 2116-30.

14. Song DH, Ko GH, Lee JH, et al. Myoferlin expression in non-small cell lung cancer: prognostic role and correlation with VEGFR-2 expression. Oncol Lett 2016; 11: 998-1006.
15. Kumar B, Brown NV, Swanson BJ, et al. High expression of myoferlin is associated with poor outcome in oropharyngeal squamous cell carcinoma patients and is inversely associated with HPV-status. Oncotarget 2016; 7: 18665-77.

16. Wang WS, Liu XH, Liu LX, et al. iTRAQ-based quantitative proteomics reveals myoferlin as a novel prognostic predictor in pancreatic adenocarcinoma. J Proteomics 2013; 91: 453-65.

17. Nishimura Y, Watanabe J, Jobo T, Hattori M, Arai T, Kuramoto H. Cytologic scoring of endometrioid adenocarcinoma of the endometrium. Cancer 2005; 105: 8-12. 